\title{
The influence of a fluid-porous interface on solar pond stability
}

\author{
A.A. Hilla,*, M. Carr ${ }^{1}$ \\ ${ }^{a}$ Department of Applied Sciences, University of the West of England, Bristol, \\ $B S 161 Q Y, U K$ \\ ${ }^{b}$ School of Mathematics and Statistics, University of St Andrews, St Andrews, \\ KY16 9SS, UK
}

\begin{abstract}
The linear instability of the gradient zone of a solar pond containing a fluidporous interface is investigated. It is found that the gradient zone can retain the same stability for lower values of the solute Rayleigh number with the introduction of a porous material compared with a purely fluid layer, whilst maintaining the same lower convective zone temperature.

Interestingly, it is also shown that for certain parameter values the penetration of a porous medium into the gradient zone can cause the temperature of the lower convective zone to rise. However, for certain parameter ranges, when the fluid-porous interface is towards the top of the gradient zone, the solar pond can become highly unstable.
\end{abstract}

Keywords: solar pond, fluid/porous interface, linear instability

\section{Introduction}

Solar ponds are pools of saltwater which can collect and store solar thermal energy through the stabilizing effect of a salt gradient. When a normal pool of water is heated by the absorption of solar radiation, the warmer water rises to the surface and loses its heat to the atmosphere. The net result is that the pond water remains at nearly atmospheric temperature. In a solar pond a salt gradient acts to prevent convective motions that would otherwise

\footnotetext{
*Corresponding author. Tel.: +44 1173283653 .

Email address: Antony.Hill@uwe.ac.uk (A.A. Hill)
} 
redistribute the absorbed solar energy, which can then be trapped and stored. Avoiding the onset of convection is, therefore, crucial in the maintenance of a solar pond.

These ponds occur naturally, e.g. Solar Lake, Israel [4], or can be artificially created to generate heat and electricity, e.g. El Paso solar pond, Texas [19]. The temperature within a solar pond typically ranges from $20^{\circ} \mathrm{C}$ at the top to $90^{\circ} \mathrm{C}$ at the bottom.

With regards to structure, a solar pond typically consists of three welldefined zones, namely the upper and lower convective zones (UCZ and LCZ) where the salinity and temperature can be considered to be uniform, and the gradient zone (GZ) where there are salt and temperature gradients, see Figure 1.

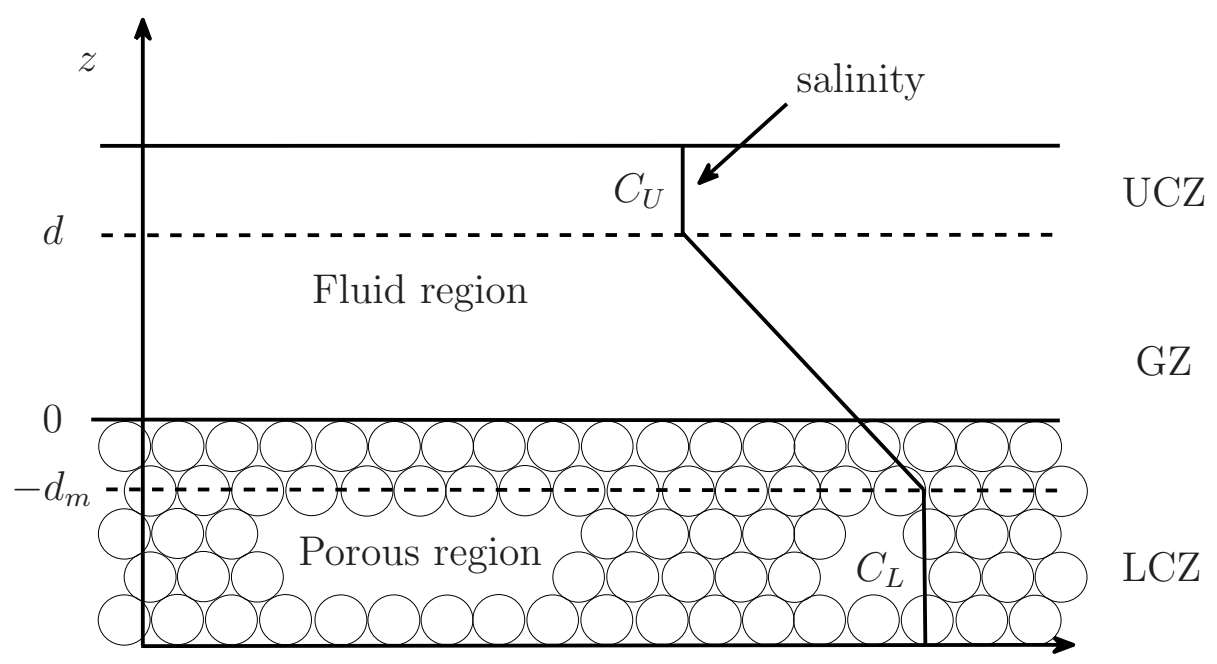

Figure 1: Schematic representation of the structure of a solar pond, with a fluid-porous interface within the gradient zone (GZ).

The main stability problem of the pond is, therefore, to maintain the non-convectivity of the GZ region.

There has been extensive experimental and theoretical research into modelling solar ponds (cf. [2, 8, 16, 21] and the references contained within). More recent work has studied the introduction of porous media to improve stability $[17,22]$ and with imposed downward convection $[9,15,18]$. By 
introducing a porous medium into the bottom of the pond, this paper investigates what influence a GZ fluid-porous interface (see Figure 1) has on solar pond stability.

We note that, in a wider context, this is the first instance in which the stability of a double diffusive convection problem with a fluid-porous interface and the absorption of radiation has been studied in the current literature.

In this paper the modelling of the fluid-porous system is addressed using a two-layer approach, where the governing equations in the separate fluid and porous regions are coupled by appropriate interfacial boundary conditions, see e.g. [3, 11, 12, 24, 25].

An alternative modelling stance is to assume a one-domain approach, which couples the porous media flow with the viscous fluid flow in a single form of equations with highly discontinuous coefficients $[10,13,14]$. However, this approach is mainly used when modelling highly porous materials, where the Darcy-Brinkman equation is more appropriate, cf. [20].

The stability results, which involve determining eigenvalues and eigenfunctions, are derived numerically using the Chebyshev tau-QZ method [5], which is a spectral method coupled with the QZ algorithm. All numerical results were checked by varying the number of polynomials to verify convergence.

\section{Formation of the problem}

To explore the behaviour of a fluid-porous interface on the efficiency and stability of a solar pond, let us consider a gradient zone (GZ) contained in the region $\left\{(x, y) \in \mathbb{R}^{2}\right\} \times\left\{z \in\left(-d_{m}, d\right)\right\}$, with concentrations $C_{L}$ in the lower convective zone (LCZ) and $C_{U}<C_{L}$ in the upper convective zone (UCZ). The gradient zone (GZ) is assumed to contain a fluid/porous interface such that the fluid occupies the region $\left\{(x, y) \in \mathbb{R}^{2}\right\} \times\{z \in(0, d)\}$ which overlies a homogeneous porous medium occupying the layer $\left\{(x, y) \in \mathbb{R}^{2}\right\} \times\{z \in$ $\left.\left(-d_{m}, 0\right)\right\}$. The interface between the saturated porous medium and the fluid layer is at $z=0$. A schematic representation of this configuration is given in Figure 1.

We assume that the density of the fluid $\rho$ has a linear temperature and concentration dependence of the form

$$
\rho(T, C)=\rho_{0}\left(1-\alpha_{t}\left(T-T_{0}\right)+\alpha_{c}\left(C-C_{0}\right)\right),
$$


where $T$ is temperature, $C$ is concentration, $\rho_{0}, T_{0}$ and $C_{0}$ are reference density, external temperature and concentration values respectively, and $\alpha_{t}$ and $\alpha_{c}$ are the coefficients of thermal and solutal expansion respectively. Field studies $[4,6]$ show that such an assumption is justified over a certain range of temperature and concentration values which vary with season and local forcing. In this study, parameter values are chosen that reflect those given in Cohen et al. for which a linear approximation is appropriate. The governing equations in the fluid layer are given by the Boussinesq approximation of the Navier-Stokes flow equation, the incompressibility condition and the balance of energy and solute, namely

$$
\begin{aligned}
\rho_{0}\left(\frac{\partial \mathbf{v}}{\partial t}+(\mathbf{v} \cdot \nabla) \mathbf{v}\right)= & -\nabla p+\mu \nabla^{2} \mathbf{v} \\
\nabla \cdot \mathbf{v}= & -\mathbf{k} g \rho_{0}\left(1-\alpha_{t}\left(T-T_{0}\right)+\alpha_{c}\left(C-C_{0}\right)\right) \\
\frac{\partial T}{\partial t}+\mathbf{v} \cdot \nabla T= & \kappa_{f} \nabla^{2} T+\frac{\dot{q}}{\left(\rho_{0} c_{p}\right)_{f}}, \\
\frac{\partial C}{\partial t}+\mathbf{v} \cdot \nabla C= & \kappa_{c} \nabla^{2} C .
\end{aligned}
$$

In these equations $\mathbf{v}=(u, v, w), p, t$ and $\mu$ are velocity, pressure, time and dynamic viscosity respectively. $\mathbf{k}=(0,0,1), \kappa_{f}=\kappa_{f}^{\prime} /\left(\rho_{0} c_{p}\right)_{f}$ and $\kappa_{f}^{\prime}, \kappa_{c}$, $g$ and $c_{p}$ are the thermal diffusivity, solutal diffusivity, acceleration due to gravity and specific heat at a constant pressure respectively.

As the solar energy is transmitted into the solar pond it is partially absorbed along its trajectory. The source term $\dot{q}$ in (3) represents the rate of energy generation per unit volume in the fluid layer due to this absorption of solar energy. Using Lambert's law we have

$$
\dot{q}=q(d) \beta_{f} e^{\beta_{f}(z-d)} \quad \text { for } \quad z \in[0, d],
$$

where $q(d)$ is the heat flux due to solar radiation at the upper boundary of the fluid layer (i.e. at $z=d$ ). The extinction coefficient $\beta_{f}$ takes into account the transparency of the medium, for example see $[7,16]$.

The governing equations in the porous layer are given by Darcy's equation (see $[20,25]$ ), the incompressibility condition and the balance of energy and 
solute, namely

$$
\begin{aligned}
& \frac{\mu}{K} \mathbf{v}^{m}=-\nabla p^{m}-\mathbf{k} g \rho_{0}\left(1-\alpha_{t}\left(T^{m}-T_{0}\right)\right. \\
& \nabla \cdot \mathbf{v}^{m}=\left.+\alpha_{c}\left(C^{m}-C_{0}\right)\right), \\
& \frac{\left(\rho_{0} c_{p}\right)_{m}}{\left(\rho_{0} c_{p}\right)_{f}} \frac{\partial T^{m}}{\partial t}+\mathbf{v}^{m} \cdot \nabla T^{m}=\kappa_{m} \nabla^{2} T^{m}+\frac{\dot{q}^{m}}{\left(\rho_{0} c_{p}\right)_{f}}, \\
& \varepsilon \frac{\partial C^{m}}{\partial t}+\mathbf{v}^{m} \cdot \nabla C^{m}=\varepsilon \kappa_{c} \nabla^{2} C^{m} .
\end{aligned}
$$

In these equations the variables $\mathbf{v}^{m}=\left(u^{m}, v^{m}, w^{m}\right), p^{m}, T^{m}, C^{m}, K$ and $\varepsilon$ are the velocity, pressure, temperature, concentration, permeability and porosity in the porous medium respectively. $\kappa_{m}=\kappa_{m}^{\prime} /\left(\rho_{0} c_{p}\right)_{f}$ and the quantities $\kappa_{m}^{\prime}$ and $\left(\rho_{0} c_{p}\right)_{m}$ are defined in terms of the fluid and solid components of the porous medium, such that $Q_{m}=\varepsilon Q_{f}+(1-\varepsilon) Q_{s}$, where $Q_{m}=\kappa_{m}^{\prime}$ or $\left(\rho_{0} c_{p}\right)_{m}$. Subscripts $f$ and $s$ denote the fluid and solid components of the porous media respectively.

Using (5), the heat flux due to solar radiation at the upper boundary of the porous layer (i.e. at the interface $z=0$ ) is $q(d) e^{-\beta_{f} d}$. Thus, using Lambert's law, the rate of energy generation per unit volume in the porous layer $\dot{q}^{m}$ is given by

$$
\dot{q}^{m}=q(d) \beta_{m} e^{\beta_{m} z-\beta_{f} d} \quad \text { for } \quad z \in\left[-d_{m}, 0\right],
$$

where $\beta_{m}$ is the extinction coefficient of the porous medium.

At the interface $z=0$, the continuity of normal velocity, temperature, concentration, heat flux and solute flux respectively yield

$$
\begin{aligned}
w & =w^{m}, \quad T=T^{m}, \quad C=C^{m}, \\
\kappa_{f}^{\prime} \frac{\partial T}{\partial z} & =\kappa_{m}^{\prime} \frac{\partial T^{m}}{\partial z}, \quad \kappa_{c} \frac{\partial C}{\partial z}=\varepsilon \kappa_{c} \frac{\partial C^{m}}{\partial z} .
\end{aligned}
$$

The remaining boundary conditions at $z=0$ are the continuity of normal stresses

$$
-p+2 \mu \frac{\partial w}{\partial z}=-p^{m}
$$

and the experimentally suggested Beavers-Joseph condition [1]

$$
\frac{\partial u}{\partial z}=\frac{\alpha}{\sqrt{K}}\left(u-u^{m}\right), \quad \frac{\partial v}{\partial z}=\frac{\alpha}{\sqrt{K}}\left(v-v^{m}\right),
$$


where $\alpha$ is a constant that depends on the porous media. Boundary condition (12) does not state that the pressure is discontinuous at the respective interfaces. In the porous medium, we interpret the pressure as a pore-averaged pressure and so condition (12) is consistent with continuous pressure in the fluid on the microscopic level.

At the upper boundary of the gradient zone $(z=d)$ we prescribe the conditions

$$
w=0 ; \quad \frac{\partial^{2} w}{\partial z^{2}}=0 ; \quad C=C_{U} ; \quad \kappa_{f}^{\prime} \frac{\partial T}{\partial z}=-h_{d}\left(T(d)-T_{0}\right),
$$

where $T_{0}$ is the external temperature and $h_{d}$ is the natural convection heat transfer coefficient $[7,16]$. The term involving heat flux reflects the fact that the heat flux from the GZ must be equal to the heat transferred to the surface by convection. $T(d)$ is the temperature at the top of the GZ and $T_{0}$ is the temperature at the surface. At the lower boundary of the gradient zone $\left(z=-d_{m}\right)$ we prescribe the conditions

$$
w^{m}=0 ; \quad C^{m}=C_{L} ; \quad \kappa_{m}^{\prime} \frac{\partial T^{m}}{\partial z}=-q_{L C Z},
$$

where $q_{L C Z}$ is the heat flux from the lower storage zone. Since the bottom of the pond is assumed to be insulated we have $q_{L C Z}=q_{a b s}-q_{e x t}$, where $q_{a b s}$ and $q_{\text {ext }}$ are the total heat absorbed and extracted in the LCZ per unit area, respectively. For simplicity it is assumed (only for the derivation of $q_{a b s}$ ) that the LCZ is of infinite depth, such that

$$
q_{a b s}=\int_{-\infty}^{-d_{m}} \dot{q}^{m}(z) d z=q(d) e^{-\beta_{m} d_{m}-\beta_{f} d} .
$$

Hence, representing the extracted heat flux as a fraction $f$ of the absorbed heat flux, $q_{L C Z}$ is given by

$$
q_{L C Z}=(1-f) q_{a b s}=(1-f) q(d) e^{-\beta_{m} d_{m}-\beta_{f} d} .
$$

The governing equations (1)-(10) and boundary conditions (11)-(15) admit a non time-dependent steady state solution in which the velocity field is 
zero (corresponding to no fluid flow) and

$$
\begin{aligned}
\bar{T}(z)= & T_{0}+q(d)\left(\frac{1}{h_{d}}+\frac{1}{\kappa_{f}^{\prime} \beta_{f}}\right)+\frac{f q(d)}{\kappa_{f}^{\prime}}\left(z-\frac{\kappa_{f}^{\prime}}{h_{d}}-d\right) e^{-\beta_{m} d_{m}-\beta_{f} d} \\
& -\frac{q(d)}{\kappa_{f}^{\prime} \beta_{f}} e^{\beta_{f}(z-d)} \\
\bar{C}(z)= & C_{U}+\frac{\varepsilon\left(C_{L}-C_{U}\right)}{\varepsilon d+d_{m}}(d-z), \\
\nabla \bar{p}= & -\mathbf{k} g \rho_{0}\left(1-\alpha_{t}\left(\bar{T}-T_{0}\right)+\alpha_{c}\left(\bar{C}-C_{0}\right)\right),
\end{aligned}
$$

on $z \in[0, d]$ and

$$
\begin{aligned}
\bar{T}^{m}(z)= & T_{0}+q(d)\left(\frac{1}{h_{d}}+\frac{1}{\kappa_{f}^{\prime} \beta_{f}}\right)+\frac{f q(d)}{\kappa_{f}^{\prime}}\left(\frac{\kappa_{f}^{\prime}}{\kappa_{m}^{\prime}} z-\frac{\kappa_{f}^{\prime}}{h_{d}}-d\right) e^{-\beta_{m} d_{m}-\beta_{f} d} \\
& +q(d) e^{-\beta_{f} d}\left(\frac{1}{\kappa_{m}^{\prime} \beta_{m}}-\frac{1}{\kappa_{f}^{\prime} \beta_{f}}-\frac{e^{\beta_{m} z}}{\kappa_{m}^{\prime} \beta_{m}}\right) \\
\bar{C}^{m}(z)= & C_{U}+\frac{C_{L}-C_{U}}{\varepsilon d+d_{m}}(\varepsilon d-z), \\
\nabla \bar{p}^{m}= & -\mathbf{k} g \rho_{0}\left(1-\alpha_{t}\left(\bar{T}^{m}-T_{0}\right)+\alpha_{c}\left(\bar{C}^{m}-C_{0}\right)\right)
\end{aligned}
$$

on $z \in\left[-d_{m}, 0\right]$, with the overbar denoting the steady state.

To illustrate the behaviour of the steady state to those profiles without any porous material, Figure 2 gives some representative temperatures at the lower boundary of the gradient zone (i.e. $\bar{T}^{m}\left(-d_{m}\right)$ ) for a specific physical system. The physical parameters are taken to be $T_{0}=20^{\circ} \mathrm{C}$, $\kappa_{f}^{\prime}=0.6 W^{-1 \circ} \mathrm{C}^{-1}, \kappa_{m}^{\prime}=0.8 W m^{-1 \circ} \mathrm{C}^{-1}, h_{d}=100 W^{-2 \circ} \mathrm{C}^{-1}, q(d)=$ $50 \mathrm{Wm}^{-2}$ and $\beta_{f}=0.2 \mathrm{~m}^{-1},[7,16]$. The combined fluid/porous depth of the layer is taken to be $1 m$, which corresponds to $d+d_{m}=1$. The nondimensional parameter

$$
\hat{d}=\frac{d}{d+d_{m}},
$$

is the fraction of the total layer depth taken up by the fluid. Thus, $\hat{d}=1$ corresponds to a layer consisting solely of fluid material, with $\hat{d}=0$ corresponding to an entirely porous layer. The values for the extinction coefficient of the porous material $\beta_{m}$ are taken from [9]. 

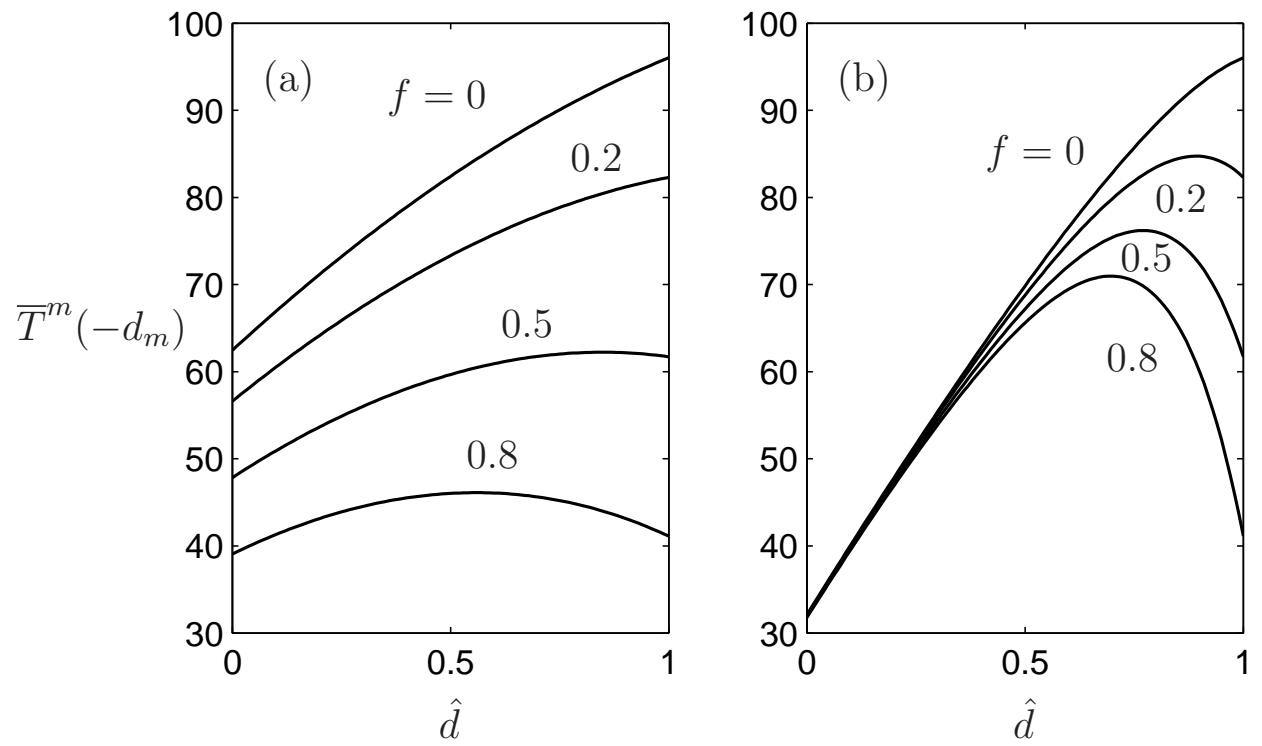

Figure 2: Representation of steady state temperature $\bar{T}^{m}\left(-d_{m}\right)$ against the fraction of the gradient zone taken by the fluid $\hat{d}$. The extracted heat flux in the storage zone (taken as a fraction $f$ of the absorbed heat flux) is taken to be $0,0.2,0.5$ and 0.8 , with (a) $\beta_{m}=0.7$ and (b) $\beta_{m}=5$. 
From Figure 2 we can see that when more heat flux is extracted in the storage zone (i.e. $f$ is increased), $\bar{T}^{m}\left(-d_{m}\right)$ falls (for fixed $\hat{d}$ ), as one would intuitively expect.

A much more interesting effect manifests when porous material is introduced into the gradient zone. For example, for $f=0.8$ in Figure 2(b) the maximum value of $\bar{T}^{m}\left(-d_{m}\right)$ actually occurs when the bottom $30 \%$ of the gradient zone is porous material, namely $\bar{T}^{m}\left(-d_{m}\right) \approx 71^{\circ} \mathrm{C}$ at $\hat{d}=0.7$. For a solely fluid layer, $\hat{d}=1$, we have $\bar{T}^{m}\left(-d_{m}\right) \approx 41^{\circ} \mathrm{C}$. This means that the introduction of the porous material has resulted in a $30^{\circ} \mathrm{C}$ rise in temperature at this point of the layer. This is less pronounced in Figure 2(a) where $\beta_{m}=0.7$ (as opposed to $\beta_{m}=5$ in Figure 2(b)) and, in general, for lower values of $f$.

The only variable which changes the structure of the steady state temperature profiles significantly is the porous media extinction coefficient $\beta_{m}$, as shown in Figure 3. The remaining parameters are taken as in Figure 2 with $f=0.5$ and $\hat{d}=0.5$. As $\beta_{m}$ is increased the gradient of the temperature profile becomes sharper. At $\beta_{m}=25$ the temperature profile is essentially constant throughout the bulk of the porous layer, whereas for $\beta_{m}=0.7$ and 5 there is still a gradient. Physically, this does raise the question of whether the salt gradient would extend to the bottom of the GZ or not (when, in the model, the temperature gradient is essentially constant for a significant proportion of the GZ), suggesting possible physcial limits on the value of $\hat{d}$, i.e. how far the porous medium can penetrate into the GZ. Further experimental work would be beneficial to explore this issue. The behaviour of the model with this temperature profile type is discussed further in Section 4.

To assess the stability of the steady solution we introduce perturbations to the steady state solution, such that

$$
\begin{aligned}
\mathbf{v}=\overline{\mathbf{v}}+\mathbf{u}, \quad T=\bar{T}+\theta, \quad C=\bar{C}+\phi, \quad p=\bar{p}+\hat{p}, \\
\mathbf{v}^{m}=\overline{\mathbf{v}}^{m}+\mathbf{u}^{m}, \quad T^{m}=\bar{T}^{m}+\theta^{m}, \quad C^{m}=\bar{C}^{m}+\phi^{m}, \quad p^{m}=\bar{p}^{m}+\hat{p}^{m} .
\end{aligned}
$$

The governing equations (1)-(4) of the fluid layer are non-dimensionalised with the scalings

$$
\begin{gathered}
\mathbf{u}=\frac{\kappa_{f}}{\left(d+d_{m}\right)} \mathbf{u}^{*}, \quad \hat{p}=\frac{\mu \kappa_{f}}{\left(d+d_{m}\right)^{2}} \hat{p}^{*}, \quad \theta=T_{0} \theta^{*}, \quad \phi=\left(C_{L}-C_{U}\right) \phi^{*}, \\
\mathbf{x}=\left(d+d_{m}\right) \mathbf{x}^{*}, \quad t=\frac{\left(d+d_{m}\right)^{2}}{\kappa_{f}} t^{*} .
\end{gathered}
$$




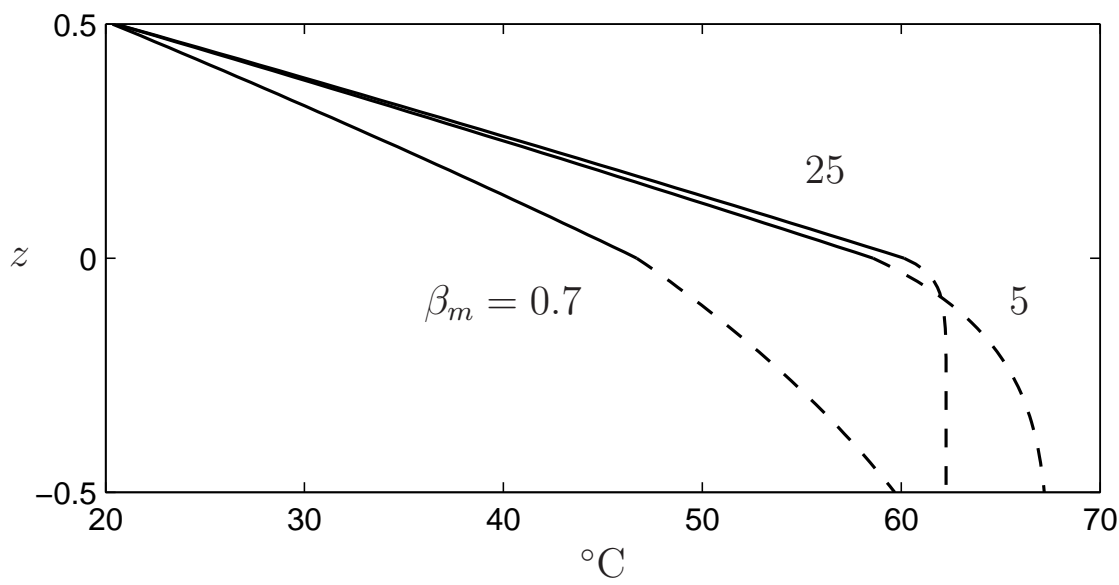

Figure 3: Steady state temperature profiles with depth $z$ against temperature in ${ }^{\circ} \mathrm{C}$. The profiles correspond to $\beta_{m}=0.7,5$ and 25 .

The porous layer scalings follow analogously from those of the fluid layer by replacing $\kappa_{f}$ with $\kappa_{m}$, with $\hat{p}^{m}=\left(\hat{p}^{m}\right)^{*} \mu \kappa_{m} / K$.

Defining the non-dimensional variables

$$
\begin{gathered}
\hat{d}=\frac{d}{d+d_{m}}, \quad Q=\frac{q(d)\left(d+d_{m}\right)}{\kappa_{f}^{\prime} T_{0}}, \quad \mu_{f}=\beta_{f}\left(d+d_{m}\right), \quad \mu_{m}=\beta_{m}\left(d+d_{m}\right), \\
\epsilon_{T}=\frac{\kappa_{f}^{\prime}}{\kappa_{m}^{\prime}}, \quad G_{m}=\frac{\left(\rho_{0} c_{p}\right)_{m}}{\left(\rho_{0} c_{p}\right)_{f}}, \quad \delta=\frac{K}{\left(d+d_{m}\right)^{2}},
\end{gathered}
$$

and substituting the perturbations and non-dimensionalised variables into (1)-(10) (and dropping the stars), we derive the systems

$$
\begin{aligned}
\operatorname{Pr}^{-1}\left(\frac{\partial \mathbf{u}}{\partial t}+(\mathbf{u} \cdot \nabla) \mathbf{u}\right) & =-\nabla \hat{p}+\nabla^{2} \mathbf{u}+\mathbf{k} R_{T}^{2} \theta-\mathbf{k} R_{S}^{2} \phi \\
\nabla \cdot \mathbf{u} & =0 \\
\frac{\partial \theta}{\partial t}+\mathbf{u} \cdot \nabla \theta & =Q F_{1}(z) w+\nabla^{2} \theta, \\
L e\left(\frac{\partial \phi}{\partial t}+\mathbf{u} \cdot \nabla \phi\right) & =\varepsilon M w+\nabla^{2} \phi
\end{aligned}
$$


in $\mathbb{R}^{2} \times(0, \hat{d}) \times(0, \infty)$, where $F_{1}(z)=e^{-\mu_{f} \hat{d}}\left(e^{\mu_{f} z}-f e^{-\mu_{m}(1-\hat{d})}\right)$ and

$$
\begin{aligned}
\mathbf{u}^{m} & =-\nabla \hat{p}^{m}+\mathbf{k} \delta \epsilon_{T} R_{T}^{2} \theta^{m}-\mathbf{k} \delta \epsilon_{T} R_{S}^{2} \phi^{m}, \\
\nabla \cdot \mathbf{u}^{m} & =0 \\
G_{m} \frac{\partial \theta^{m}}{\partial t}+\mathbf{u}^{m} \cdot \nabla \theta^{m} & =Q \epsilon_{T} F_{2}(z) w^{m}+\nabla^{2} \theta^{m} \\
L e\left(\varepsilon \frac{\partial \phi^{m}}{\partial t}+\mathbf{u}^{m} \cdot \nabla \phi^{m}\right) & =M w^{m}+\epsilon_{T} \varepsilon \nabla^{2} \phi^{m}
\end{aligned}
$$

in $\mathbb{R}^{2} \times(\hat{d}-1,0) \times(0, \infty)$, where $F_{2}(z)=e^{-\mu_{f} \hat{d}}\left(e^{\mu_{m} z}-f e^{-\mu_{m}(1-\hat{d})}\right)$ and $M=L e /(\varepsilon \hat{d}+1-\hat{d})$. The remaining non-dimensional variables are given by

$R_{T}^{2}=\frac{g \alpha_{t} T_{0}\left(d+d_{m}\right)^{3} \rho_{0}}{\mu \kappa_{f}}, \quad R_{S}^{2}=\frac{g \alpha_{c}\left(C_{L}-C_{U}\right)\left(d+d_{m}\right)^{3} \rho_{0}}{\mu \kappa_{f}}, \quad \operatorname{Pr}=\frac{\mu}{\rho_{0} \kappa_{f}}, \quad L e=\frac{\kappa_{f}}{\kappa_{c}}$,

where $R_{T}^{2}$ and $R_{S}^{2}$ are the fluid thermal and solute Rayleigh numbers, respectively, $\operatorname{Pr}$ is the Prandtl number and $L e$ is the Lewis number.

\section{Linear Instability Analysis}

To proceed with a linear instability analysis the nonlinear terms in equations (16) to (19) are discarded. Since the resulting system is linear and autonomous we may seek solutions of the form

$$
\begin{aligned}
& \mathbf{u}=\mathbf{u}(z) \mathrm{e}^{\sigma t+i\left(a_{1} x+a_{2} y\right)}, \quad \hat{p}=\hat{p}(z) \mathrm{e}^{\sigma t+i\left(a_{1} x+a_{2} y\right)}, \\
& \theta=\theta(z) \mathrm{e}^{\sigma t+i\left(a_{1} x+a_{2} y\right)}, \quad \phi=\phi(z) \mathrm{e}^{\sigma t+i\left(a_{1} x+a_{2} y\right)},
\end{aligned}
$$

where $a_{1}, a_{2} \in \mathbb{R}$ are the horizontal wavenumbers and $\sigma \in \mathbb{C}$. Analogous definitions apply to the porous layer equations (20)-(23), with superscript $m$. The growth rate $\sigma$ (noting $\sigma^{m}=\epsilon_{T} \sigma$ ) can now be used to assess whether the non time-dependent steady state is unstable. If $\operatorname{Re}(\sigma)>0$, then the perturbation will grow exponentially in time, clearly leading to linear instability.

Letting $D=d / d z$, and taking the double curl of (16) and (20) to remove the pressure terms, systems (16)-(19) and (20)-(23) become

$$
\begin{aligned}
& \left(D^{2}-a^{2}\right)^{2} w-a^{2} R_{T}^{2} \theta+a^{2} R_{S}^{2} \phi=\frac{\sigma}{P r}\left(D^{2}-a^{2}\right) w \\
& \left(D^{2}-a^{2}\right) \theta+Q F_{1} w=\sigma \theta \\
& \left(D^{2}-a^{2}\right) \phi+\varepsilon M w=\sigma L e \phi
\end{aligned}
$$


and, noting $a_{1}^{m}=a_{1}, a_{2}^{m}=a_{2}$,

$$
\begin{aligned}
& \left(D^{2}-a^{2}\right) w^{m}+a^{2} \delta \epsilon_{T} R_{T}^{2} \theta^{m}-a^{2} \delta \epsilon_{T} R_{S}^{2} \phi^{m}=0 \\
& \left(D^{2}-a^{2}\right) \theta^{m}+Q \epsilon_{T} F_{2} w^{m}=G_{m} \epsilon_{T} \sigma \theta^{m} \\
& \varepsilon\left(D^{2}-a^{2}\right) \phi^{m}+\frac{M}{\epsilon_{T}} w^{m}=\sigma L e \varepsilon \phi^{m}
\end{aligned}
$$

where $a^{2}=a_{1}^{2}+a_{2}^{2}$. The boundary conditions for system (24)-(25) at $z=\hat{d}$ are

$$
w=D^{2} w=\phi=0, \quad D \theta=-H_{d} \theta,
$$

whereas at $z=\hat{d}-1$ they are

$$
w^{m}=D \theta^{m}=\phi^{m}=0,
$$

where $H_{d}=h_{d}\left(d+d_{m}\right) / \kappa_{f}^{\prime}$. On the interface $z=0$, we have

$$
\epsilon_{T} w=w^{m}, \quad \theta=\theta^{m}, \quad \epsilon_{T} D \theta=D \theta^{m}, \quad \phi=\phi^{m}, \quad D \phi=\varepsilon D \phi^{m} .
$$

The remaining boundary conditions on the interface come from (13) (where $(13)_{1}$ and $(13)_{2}$ are differentiated with respect to $x$ and $y$, combined using (2) and (7), and then non-dimensionalised) to give

$$
\sqrt{\delta} D^{2} w=\alpha\left(D w-\epsilon_{T}^{-1} D w^{m}\right)
$$

and (12) (where (12) is differentiated with respect to $x$ and $y$ separately, combined using (1), (2), (6) and (7), and then non-dimensionalised) to give

$$
\frac{\sigma}{\operatorname{Pr}} D w-\left(D^{2}-a^{2}\right) D w+2 a^{2} D w=\frac{1}{\epsilon_{T} \delta} D w^{m} .
$$

System (24)-(25) constitues a generalised eigenvalue problem for $\sigma$, which was solved using the Chebyshev tau-QZ method [5]. The critical thermal Rayleigh number is located by varying $R_{T}^{2}$ to find $\operatorname{Re}(\sigma)=0$ whilst minimising over the wavenumber $a^{2}$. The numerical results are presented in $\S 4$.

\section{Numerical results}

We now solve the eigenvalue problem (24)-(25) by means of a $D^{2}$ Chebyshev tau method. The details are similar to those given by Dongarra et al. [5]. Equation $(24)_{1}$ is written as two second-order equations and we solve 
equations (24)-(25), not as a fourteenth-order system, but as seven secondorder equations. The numerical results have been checked by varying the number of polynomials to verify convergence.

Taking physically realistic values $[7,9,16]$, the parameters, unless stated otherwise, are fixed at $\hat{d}=0.7, H_{d}=150, Q=4, \mu_{f}=0.2, \mu_{m}=5, \epsilon_{T}=0.7$, $f=0.5$ and $R_{S}^{2}=10000$.

Figure 4 shows the effect of $Q$ on the stability of the solar pond by giving (a) the neutral curves at representative parameter values and (b) the corresponding temperature in the $\mathrm{LCZ}, \bar{T}_{L C Z}$, (i.e. the steady state temperature at the bottom of the GZ). To aid in interpretation, the external temperature $T_{0}$ is assumed to be $20^{\circ} \mathrm{C}$, so that, in dimensional form, $\bar{T}_{L C Z}=\bar{T}\left(-d_{m}\right)$ is given in ${ }^{\circ} \mathrm{C}$.
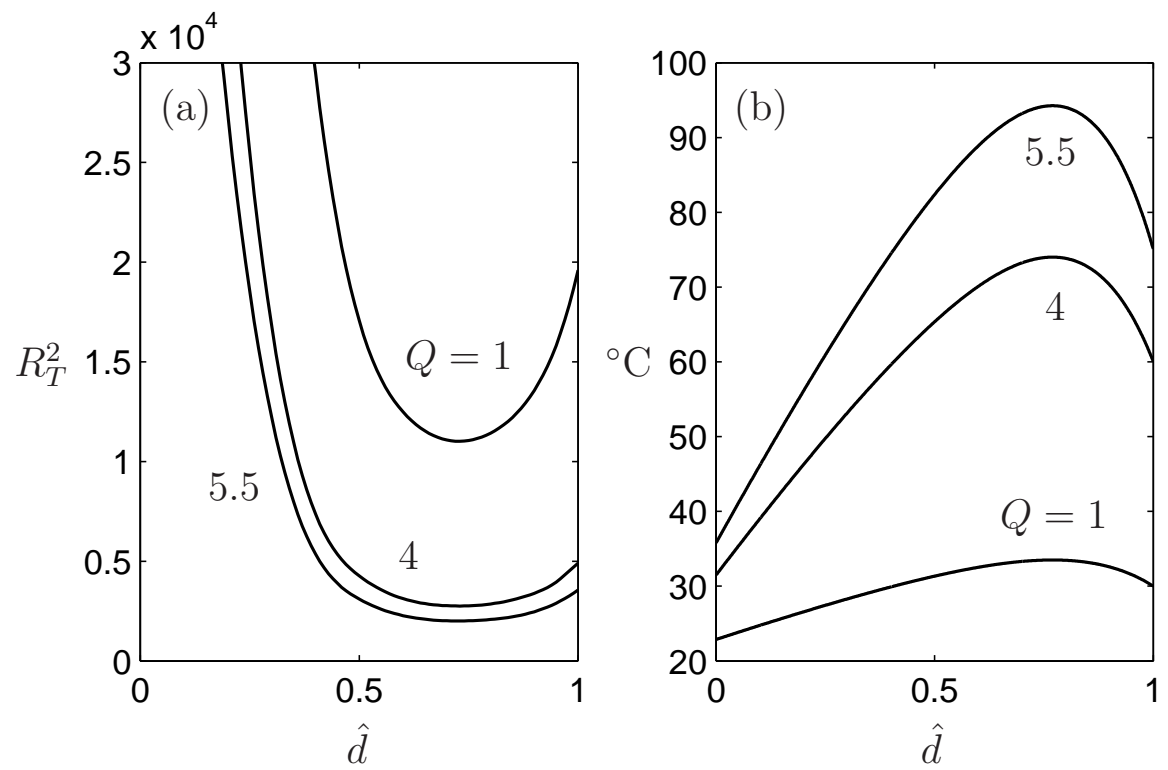

Figure 4: Graph (a) gives a visual representation of the linear instability thresholds for $Q=1,4$ and 5.5, with critical thermal Rayleigh number $R_{T}^{2}$ plotted against the fraction of the gradient zone taken by the fluid $\hat{d}$. Graph (b) gives the corresponding steady state temperature $\bar{T}_{L C Z}$ in ${ }^{\circ} \mathrm{C}$ against $\hat{d}$.

As one would intuitively expect, if the GZ layer depth is fixed, an increase in $Q$ (corresponding to an increase in the heat flux at the top of the solar pond) causes the pond to become more unstable. Interestingly, though, as the porous medium enters the GZ (i.e. as $\hat{d}$ decreases from 1 ) we can see that the 
system becomes more unstable before re-stabilizing. The corresponding LCZ temperature $\bar{T}_{L C Z}$ follows an analogous reciprocal pattern, increasing as $\hat{d}$ is reduced from 1 before decreasing. As $\hat{d} \rightarrow 0$, under the stated parameters, the pond becomes highly stable, although value of $\bar{T}_{L C Z}$ is greatly reduced.

For $Q=4$ and $5.5, \bar{T}_{L C Z}$ takes a maximum value at approximately $\hat{d}=$ 0.77 , namely $74^{\circ} \mathrm{C}$ and $94^{\circ} \mathrm{C}$. Without the presence of the porous media (i.e. $\hat{d}=1)$ the corresponding temperatures are $60^{\circ} \mathrm{C}$ and $75^{\circ} \mathrm{C}$.

Figure 5 shows the neutral curves for a variation of depth ratio $\hat{d}$ values, with critical thermal Rayleigh number $R_{T}^{2}$ plotted against solute Rayleigh number $R_{S}^{2}$.

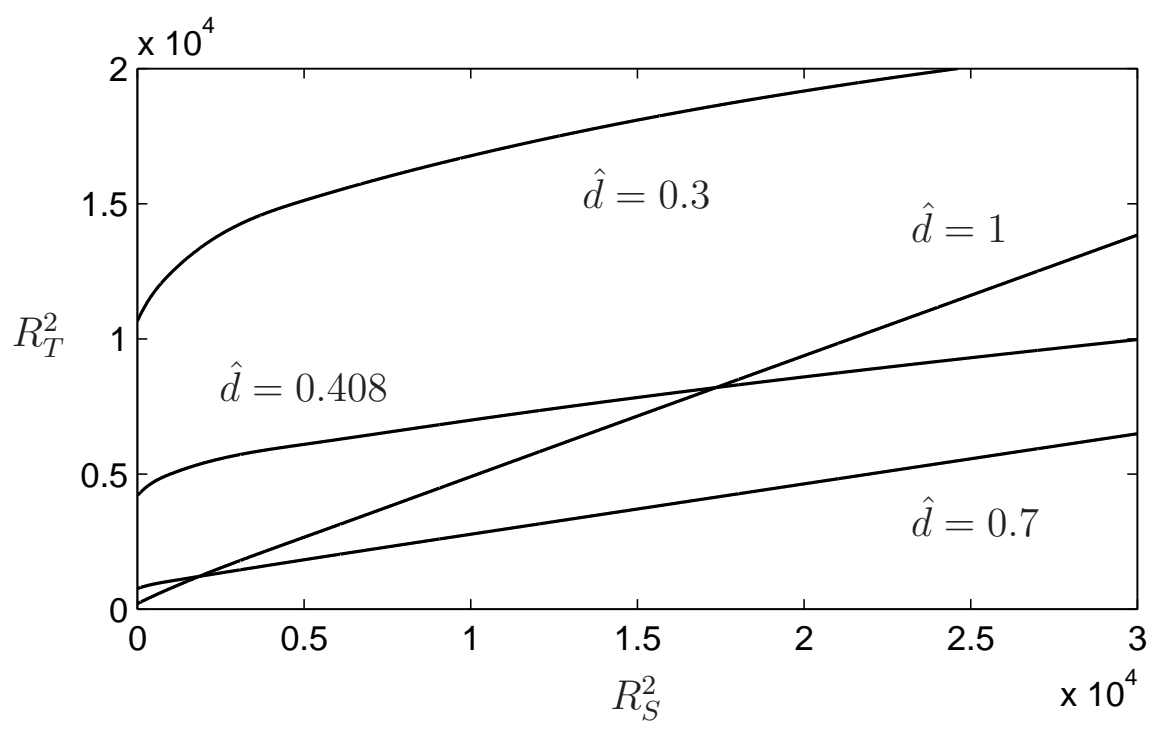

Figure 5: Visual representation of the linear instability thresholds for $\hat{d}=0.3,0.408,0.7$ and 1, with critical thermal Rayleigh number $R_{T}^{2}$ plotted against solute Rayleigh number $R_{S}^{2}$.

From Figure 5 we can see, as would be physically expected, that an increase in $R_{S}^{2}$ causes the pond to become more stable. Since $\bar{T}_{L C Z}$ is independent of $R_{S}^{2}$, the $\bar{T}_{L C Z}$ value corresponding to each $\hat{d}$ can be seen from Figure $3 \mathrm{~b}$ (for $Q=4$ ).

The highly interesting case is to compare a solely fluid layer with a fluidporous system which has the same $\bar{T}_{L C Z}$ i.e. the LCZ is at the same tempertaure with/without the porous medium. In Figure 5 this is given at $\hat{d}=1$ and $\hat{d}=0.408$ for the solely fluid and fluid-porous system, respectively. We 
can see that for lower values of the solute Rayleigh number the neutral curve for $\hat{d}=0.408$ is less unstable than for $\hat{d}=1$. Although, there is a critical value of $R_{S}^{2}(\approx 17360)$ for which the solely fluid layer becomes more stable.

This result demonstrates that, in the presence of a fluid-porous interface, a solar pond can retain the same stability for lower values of the solute Rayleigh number than a purely fluid layer, whilst maintaining the same lower convective zone temperature.

In Figure 4a it was observed that as $\hat{d} \rightarrow 0$, the pond becomes highly stable. This result was for the porous medium extinction coefficient $\mu_{m}=5$ and is repeated for all data collected for $\mu_{m} \leq 15$. Figure 6 demonstrates the behaviour of the neutral curves at representative values of $\mu_{m}$ under and over 15 .

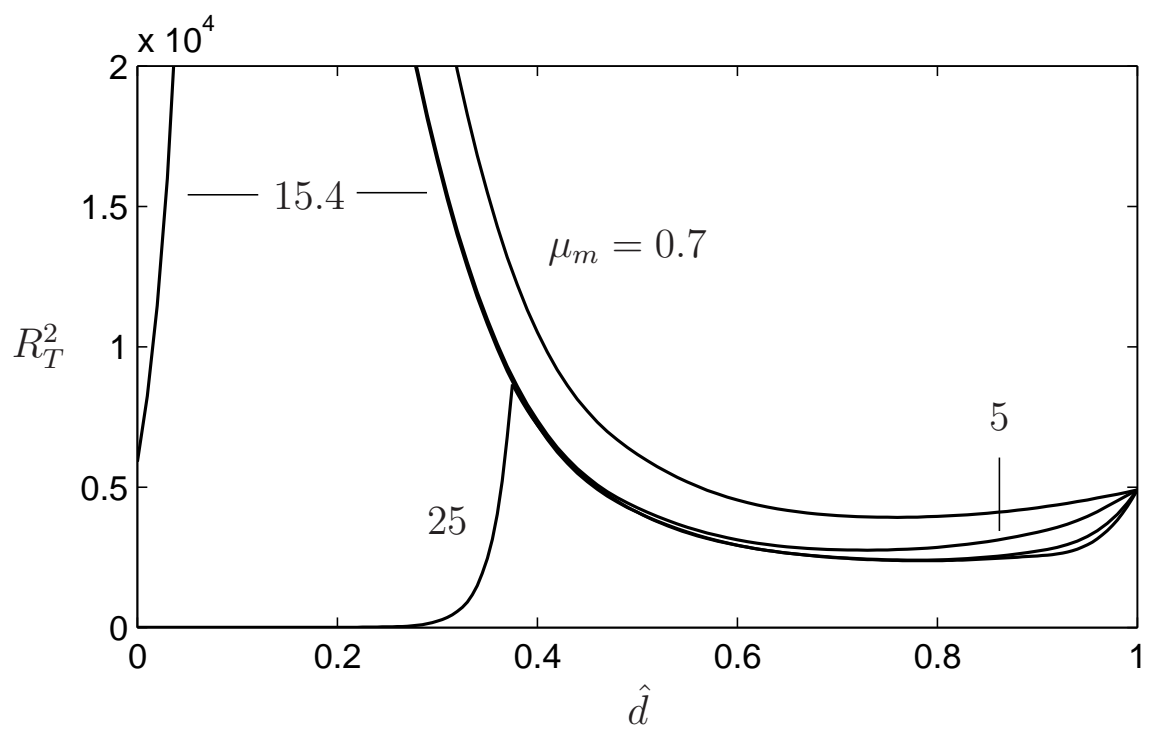

Figure 6: Visual representation of the linear instability thresholds for $\mu_{m}=0.7,5,15.4$ and 25 , with critical thermal Rayleigh number $R_{T}^{2}$ plotted against the fraction of the gradient zone taken by the fluid $\hat{d}$.

For all values of $\mu_{m}$ the neutral curves behave similarly for $\hat{d} \geq 0.4$. However, for $\mu_{m}=15.4$ there is a distinct region of instability (where the onset of convection propagates primarily in the porous region) that becomes present for $\hat{d} \leq 0.05$ i.e. where the layer is $\geq 95 \%$ porous material. As $\mu_{m}$ is increased to 25 , this region expands considerably, with the GZ being entirely unstable for lower values of $\hat{d}$. 
However, as discussed in Section 2, the validity of the model for such high values of $\mu_{m}$, where the steady state temperature profiles are essentially constant for the bulk of the porous material, is unclear. Coupled with the highly unstable nature demonstrated in Figure 6 this strongly indicates that the model validity is more substantial for higher values of $\hat{d}$, i.e. where the porous material only penetrates into the lower sections of the GZ.

\section{Conclusions}

Solar ponds capture and store solar radiation through the stabilizing effect of a salt gradient. They typically have a three layer structure, with the upper and lower layers containing roughly constant temperature and salt profiles, with a gradient zone (GZ) between them. This makes the GZ essentially a double-diffusive layer of salt and temperature. Maintaining the stability of the GZ is, therefore, crucial in its functionality. There has been extensive theroretical and experimental research conducted on solar pond modelling, with a recent development $[17,22,9,15,18]$ being the introduction of porous material.

In the present article we have analyzed the instability of the GZ of a solar pond, where there is a fluid layer overlying a porous material, with the fluid-porous interface falling within the GZ.

The results indicate that a fluid-porous pond may retain the same stability for lower solute Rayleigh numbers as compared to a purely fluid pond, whilst retaining the same lower convective zone temperature.

It is also shown that the lower convective zone temperature may also be increased by the introduction of porous material, although this does, as one would intuitively expect, result in more instability.

Although the GZ is shown to be highly unstable for high values of the porous extinction coefficient (where the bulk of the medium is porous material) it is suggested, by studying the steady state temperature profiles, that the model may not be suitable for such regions due to the essentially constant behavior of the steady state temperature in the assigned GZ.

One major issue in the design and installation of man made solar ponds is what material to use for the liner [23]. The work presented here shows that the presence of a porous material will affect the stability of a solar pond. Due to the modelling deficiencies identified above, however, a detailed investigation of the characteristics of the porous material are beyond the scope of the paper and as such are left for future study. 


\section{References}

[1] Beavers GS, Joseph DD. Boundary conditions at a naturally permeable wall. J. Fluid Mech. 1967; 30: 197-207.

[2] Ben Mansour R, Nguyen CT, Galanis N. Numerical study of transient heat and mass transfer and stability in a salt-gradient solar pond. Int. J. Thermal Sci. 2004; 43: 779-790.

[3] Chandesris M, Jamet D. Jump Conditions and Surface-Excess Quantities at a Fluid/Porous Interface: A Multi-scale Approach. Trans. Porous Media 2009; 78: 419-438.

[4] Cohen Y, Krumbein WE, Goldberg M, Shilo M. Solar Lake (Sinai). 1. Physical and chemical limnology. Limnology and Oceanography 1977; 22: 597-608.

[5] Dongarra JJ, Straughan B, Walker DW. Chebyshev tau-QZ algorithm methods for calculating spectra of hydrodynamic stability problems. Appl. Numer. Math. 1996; 22: 399-434.

[6] Eckstein, Y. Physico-Chemical Limnology and Geology of a Meromictic Pond on the Red Sea Shore. Limn. \& Oceanogr. 1970; 15: 363-372.

[7] Giestas M, Pina H, Joyce A. The influence of radiation absorption on solar pond stability. Int J. Heat Mass Transfer 1996; 39: 3873-3885.

[8] Giestas M, Pina H, Milhazes JP, Tavares C. Solar pond modeling with density and viscosity dependent on temperature and salinity. Int J. Heat Mass Transfer 2009; 52: 2849-2857.

[9] Hadim A, Burmeister LC. Conceptual design of a downward-convecting solar pond filled with a water saturated, porous medium. J. Solar Energy Eng. 1992; 114: 240-245.

[10] Hill AA, Carr M. Nonlinear stability of the one-domain approach to modelling convection in superposed fluid and porous layers. Proc. Roy. Soc. A 2010; 466: 2695-2705.

[11] Hill AA, Carr M. Sharp global nonlinear stability for a fluid overlying a highly porous material. Proc. Roy. Soc. A 2010; 466: 127-140. 
[12] Hill AA, Straughan B. Global stability for thermal convection in a fluid overlying a highly porous material. Proc. Roy. Soc. A 2009; 465: 207217.

[13] Hirata SC, Goyeau B, Gobin D, Carr M, Cotta RM. Linear stability of natural convection in superposed fluid and porous layers: influence of the interfacial modelling. Int. J. Heat Mass Transfer 2007; 50: 1356-1367.

[14] Hirata SC, Goyeau B, Gobin D. Stability of thermosolutal natural convection in superposed fluid and porous layers. Trans. Porous Media 2009; 78: $525-536$.

[15] Horng C, Sheu TS, Hwang BH, Wan CJ, Liu PC. Temperature Distribution in a Porous Medium Subjected to Solar Radiative Incidence and Downward Flow. WHAMPOA - An Interdisciplinary Journal 2006; 50: $185-194$.

[16] Kaffel Rebaï L, Mojtabi AK, Safi MJ, Mohamad AA. A linear stability study of the gradient zone of a solar pond. J. Solar Energy Eng. 2006; 128: 383-393.

[17] Karim C, Slim Z, Kais C, Jomâa SM, Akbarzadeh A. Experimental study of the salt gradient solar pond stability. Solar Energy 2010; 84: 2431 .

[18] Liu P. Temperature distribution in a porous medium subjected to solar radiative incidence and downward flow: convective boundaries. J. Sol. Energy Eng. 2003; 125: 190-194.

[19] Lu H, Swift AHP. El Paso Solar Pond. J. Sol. Energy Eng. 2001; 123: 178.

[20] Nield DA, Bejan A. Convection in porous media. 3rd ed. Springer-Verlag; 2006.

[21] Ouni M, Guizani A, Lu H, Belghith A. Simulation of the control of a salt gradient solar pond in the south of Tunisia. Solar Energy 2003; 75: 95-101.

[22] Shi Y, Yin F, Shi L, Sun W, Li N, Liu H. Effects of porous media on thermal and salt diffusion of solar pond. Applied Energy 2011; 88: 24452453. 
[23] Silvaa, G, Almanza, R. Use of clays as liners in solar ponds. Solar Energy 2009; 83: 905-919.

[24] Straughan B. Stability and wave motion in porous media. Applied mathematical sciences series, vol. 165. Springer-Verlag; 2008.

[25] Vafai K. Handbook of porous media. 2nd ed. Taylor and Francis; 2005. 